九州大学学術情報リポジトリ

Kyushu University Institutional Repository

\title{
CHATTERING ATTENUATION SLIDING MODE APPROACH FOR NONLINEAR SYSTEMS
}

Nemati, Hamidreza

Department of Aeronautics and Astronautics, Kyushu University

Bando, Ma i

Department of Aeronautics and Astronautics, Kyushu University

HOKAMOTO, Shinji

Department of Aeronautics and Astronautics, Kyushu University

ht tp://hdl. handle. net/2324/4479597

出版情報: Asian Journal of Control. 19 (4), pp.1519-1531，2017-07. Chinese Automatic Control Society

バージョン :

権利関係 : 


\title{
CHATTERING ATTENUATION SLIDING MODE APPROACH FOR NONLINEAR SYSTEMS
}

\begin{abstract}
This study introduces a new robust nonlinear control scheme based on the theory of nonsingular terminal sliding mode control (NTSMC). Since conventional NTSMC utilizes a discontinuous switching function, a significant flaw called chattering can occur. The main purpose of this study is to design a new switching function based upon Lyapunov stability in order to alleviate this drawback over time. There are many approaches to mitigate the chattering drawback in SMC such as utilizing a smooth approximation of the switching element, or employing higher order sliding mode control (HOSMC) strategy. However, the use of a continuous approximation affects the system's performance and a finite reaching time to the sliding manifold, and in HOSMC the estimation of high-order derivatives of states is usually difficult and it still exhibits chattering in the presence of parasitic dynamics. In this study by employing a new sliding manifold including a time function, the chattering is attenuated as well as keeping the robustness. Finally, a second-order nonlinear dynamical system subject to disturbance is simulated to highlight the validity and applicability of the proposed method.
\end{abstract}

KeyWords: sliding mode control, chattering attenuation, robust performance, Lyapunov stability, time-varying switching function

\section{INTRODUCTION}

Real systems exhibit natural nonlinearities that can have undesirable effects. Nonlinear systems provide a level of dynamic capabilities when dealing with parameter variations and unmodeled dynamics that linear approaches lack. Therefore, nonlinear controllers have to be designed to compensate for these conditions. Some of the most attractive features of a sliding mode control (SMC) are its inherent advantages of strong 
stability, disturbance rejection, and low sensitivity to plant parameter variations [1]. Today, application of SMC is ubiquitous to various engineering problems encountered in robotics, automation, and control [2, 3]. For a comprehensive survey of many sliding mode techniques, the reader is referred to [4] as well as the seminal work of [5].

Despite the popularity of conventional SMC (CSMC), it has a major drawback called chattering. Chattering is a condition where an infinite number of jumps (i.e., switches) of control inputs occur with a finite time interval. Basically, chattering obstructs the direct use of sliding mode approaches. Chattering can deteriorate system performance, cause mechanical wear in the system, and even lead to instability. Especially, for a flexible system with elastic appendages, chattering may destroy the system. Even though there are numerous studies on the application of SMC strategies, there is a limited extent of research regarding the suppression of chattering. This study briefly reviews a few of the chattering suppression approaches considered in the recent past.

To mitigate the chattering phenomenon in CSMC, many approaches have employed a smooth approximation of the switching element (known as a boundary layer) instead of a signum function in order to have a continuous output in the system's control law [6, 7, 8]. Even in recently published papers [9, 10, 11], boundary layer schemes are still considered as a promising method to suppress the chattering phenomenon. However, the use of a continuous approximation has two drawbacks. First, the invariance property is lost; therefore, the system's performance will depend upon the boundary layer thickness of the continuous function. Second, the continuous approximation does not assure finite reaching time to the switching manifold. To solve these problems while preserving the main advantages of CSMC, higher order sliding mode (HOSM) control was first elaborated by Emel'yanov et al.[12]. HOSM generalizes the basic sliding mode idea to act on the higher order time-derivatives of the system deviation [13] instead of the first-order derivatives used in conventional sliding modes. Recently, many researchers have relied on the HOSM approach in some applications [14, 15, 16, 17. 18. 19]. However, for HOSM, the estimation of highorder derivatives of states is usually difficult and requires complex implementation algorithms. Furthermore, Boiko et al. [20, 21] concluded that a second-order sliding mode algorithm, which is the simplest form of HOSM, still exhibits chattering in the presence of parasitic dynamics. On another front, without using higher-order derivatives, continuous control scheme has been proposed in [22]. This controller can reduce/vanish the chattering phenomenon of control inputs, even when continuous disturbance is added. However, finding proper feedback gains is not easy. Improper gains can easily induce oscillations, and moreover these feedback gains change not only the state variables and control input after the states reach the sliding surface but also the convergence speeds in both approach and sliding phases.

Basically, in the sliding phase of CSMC, a linear sliding hyperplane is selected to converge the output error to zero asymptotically. However, the tracking error cannot reach zero in a finite time. To enhance the convergence performance of the sliding mode controller, terminal sliding mode control (TSMC) has been introduced [23]. TSMC utilizes the nonlinear sliding manifold to converge the output errors to zero in a finite time. However, TSMC 
has the critical disadvantage of a singularity problem due to an existing negative fractional power in the terminal sliders. This problem can be eliminated by utilizing an inverse dynamics in the terminal sliding manifold, which has been introduced as nonsingular terminal sliding mode control (NTSMC) [24]. Much attention has been focused on various research fields involving NTSMC because of the method's finite time convergence as well as its singularity avoidance [25, 26, 27]. It should be noted that the proposed technique is applicable to any types of SMC approaches, although we chose NTSMC because of its aforementioned advantages in this paper.

In this study, a chattering attenuation NTSMC is conceived to alleviate the chattering phenomenon. The main purpose of this study is to design a sliding mode controller through the use of a new switching function in order to alleviate chattering over time. A single-input second order nonlinear dynamical system is simulated to highlight the validity and applicability of the proposed method.

The remainder of this paper is organized as follows. Section III contains a detailed analysis of SMC approaches. This section deals with the concept of finite time convergence and the existence of the chattering phenomenon in SMC. In addition, the design of a new chattering attenuation sliding mode controller is presented in Section III Section III is intended to present a methodology for the design of a new chattering attenuation strategy as well as for analyzing the performance of the designed controller. Next, Section [V] is devoted to the application of the proposed method. In this section, numerical simulations are performed to demonstrate that the chattering attenuation and performance-keeping are both accomplished in spite of the presence of disturbances. Finally, the paper concludes with some remarks.

\section{CONTROL LAW DERIVATION}

Consider a second-order nonlinear dynamical system:

$$
\ddot{x}=g(x, \dot{x})+h(x, \dot{x}) u
$$

where $x \in \mathbb{R}$ represents the system state, $g(x, \dot{x})$ and $h(x, \dot{x})>0$ are two nonlinear functions describing system dynamics, and $u \in \mathbb{R}$ is the control input to be designed. For $h(x, \dot{x})<0$, control input $u$ can be redefined as $-u$ without loss of generality. States $x$ and $\dot{x}$ are available as measured outputs, therefore the system relative degree is one over $\dot{x} \neq 0$. Although Eq. (1) and other equations in this paper have been expressed in terms of scalar forms for a nonlinear system, they can be expanded to a vector form too.

Sliding mode controllers are designed for stabilizing the system given in Eq. (1) around the desired states $x_{d}=$ 0 in this section. In the following, some SMC strategies are first summarized and then the proposed control law is introduced. The proposed controller incorporates chattering attenuation methodology into the design of the NTSMC method.

\section{II.1. Conventional SMC (CSMC)}

A conventional sliding manifold can be expressed as follows:

$$
\sigma_{\text {conv } .}(x, t)=\dot{\tilde{x}}+\lambda \tilde{x}
$$

where $\tilde{x}=x-x_{d}$ is a perturbation from the desired point.

Remark 1. According to Eq. (2), the constant gain $\lambda$ 
should be positive to assure the closed loop stability. This fact can be simply verified by

$$
\sigma_{\text {conv. }}=\dot{\tilde{x}}+\lambda \tilde{x}=0
$$

Then, the output state error through the sliding mode can be expressed as follows:

$$
\dot{\tilde{x}}=-\lambda \tilde{x} \Rightarrow \tilde{x}(t)=\tilde{x}(0) \exp (-\lambda t)
$$

Therefore, a negative value of $\lambda$ results in $\tilde{x}(t)$ moving away from zero and may lead to instability.

Remark 2. The constant gain $\lambda$ in Eq. (2) is typically limited by three factors [28]: the frequency of the lowest unmodeled structural resonant mode $\left(\nu_{R}\right)$, the largest neglected time delay $\left(T_{A}\right)$, and the sampling rate $\left(\nu_{s}\right)$ as follows:

$$
\lambda \leq \frac{2 \pi}{3} \nu_{R} \quad, \quad \lambda \leq \frac{1}{3 T_{A}} \quad, \quad \lambda \leq \frac{1}{5} \nu_{s}
$$

To guarantee the attractiveness of the sliding manifold, consider the following positive definite function of $\sigma_{\text {conv }}$. as a Lyapunov function

$$
V=\frac{1}{2} \sigma_{\text {conv. }}^{2}
$$

The time-derivative of this function can be written as follows:

$$
\dot{V}=\sigma_{\text {conv }} \frac{\partial \sigma_{\text {conv }}}{\partial t}=\sigma_{\text {conv }} .(\ddot{\tilde{x}}+\lambda \dot{\tilde{x}})
$$

For the existence of the attractive sliding mode, it is easily verified that the time-derivative of the selected Lyapunov function must satisfy $\dot{V}<0$; i.e.,

$$
\dot{V}=-\mu\left|\sigma_{\text {conv }}\right|
$$

where the parameter $\mu$ is a strictly positive constant and should be greater than the magnitude of the disturbance (Section III). Substituting Eq. (8) into Eq. (7) yields

$$
\sigma_{\text {conv. }}(\ddot{\tilde{x}}+\lambda \dot{\tilde{x}})=-\mu\left|\sigma_{\text {conv }}\right|
$$

One can rewrite Eq. (9) by means of Eq. (1) and assuming $\ddot{x}_{d}=0$ as follows:

$$
g(x, \dot{x})+h(x, \dot{x}) u+\lambda \dot{\tilde{x}}=-\mu \operatorname{sign}\left(\sigma_{\text {conv }}\right)
$$

Therefore, for the nonlinear dynamical system in Eq. (1) with the conventional switching surface in Eq. (2), if the control is designed as

$$
u=-h(x, \dot{x})^{-1}\left[\mu \operatorname{sign}\left(\sigma_{\text {conv. }}\right)+\lambda \dot{\tilde{x}}+g(x, \dot{x})\right]
$$

then the states move toward the sliding manifold and the motion is confined to it. Finally, from Eq. (4), the state errors asymptotically converge to zero.

According to Eq. (11), $\operatorname{sign}\left(\sigma_{\text {conv }}\right)$ is a signum function and can be determined as follows:

$$
\operatorname{sign}\left(\sigma_{c o n v .}\right)=\frac{\left|\sigma_{c o n v .}\right|}{\sigma_{c o n v .}}= \begin{cases}1 & \sigma_{c o n v .}>0 \\ 0 & \sigma_{c o n v .}=0 \\ -1 & \sigma_{c o n v .}<0\end{cases}
$$

As can be seen from Eq. (12), the signum function is a discontinuous function that can switch the control signal at an infinite frequency, and thereby excite the unmodeled fast dynamics or undesirable oscillations called chattering. 


\section{II.2. Chattering Attenuation Conventional SMC} (CACSMC)

Chattering is an inevitable phenomenon due to the inherent discontinuity or switching nature around manifolds. The adaptive sliding gain in [3] is undesired because the state's response abruptly has overshoot around the origin. Also, the states converge to the vicinity of the sliding manifold and it cannot assure the states to keep around the sliding manifold after convergence. On another front, higher-order sliding mode (HOSM) strategy is able to solve the chattering drawback. However, it has some disadvantages:

- In HOSM, the estimation for higher-order states is necessary and it requires usually a complicated additional subsystem.

- The convergence and robustness of the secondorder sliding mode (SOSM), which is a simplest form of HOSM, has been established using only homogeneity approach [29]. However, this approach cannot guarantee the finite time stability of HOSM.

- The system trajectory is very slow when the states are far away from the origin in SOSM [30].

- SOSM cannot endure uncertainties and disturbances that change with the system's states [31].

- Even in applying HOSM, some papers point out that chattering may still exist.

Hereby, without violating the sliding condition, an improved SMC strategy is proposed to reduce the value of the discontinuous function over time. This idea is new and it has not been considered yet.

$$
\begin{aligned}
\sigma_{C A C S M C}(x, t) & =f(t) \sigma_{\text {conv. }}(x, t) \\
& =f(t)(\dot{\tilde{x}}+\lambda \tilde{x})
\end{aligned}
$$

where $f(t)$ is a continuous time-varying function in $\mathbb{R}^{+}$. As can be clearly seen from Eq. (13), a new chattering attenuation sliding surface is expanded with a time-varying function $f(t)$ which has a pivotal role in the proposed strategy. The new proposed time-varying function has several advantages:

- Simplicity of the proposed approach.

- The proposed time-varying function can be implemented to any types of SMC algorithms.

- The proposed approach can attenuate the chattering phenomenon over time with various time-varying functions.

- The proposed approach can fix the robust performance of the controller after some definite time instants which may be designed optionally.

- The proposed approach can retain the robust performance of the controller which may be determined by a designer.

These ascertainable advantages are proved in this paper later. Let us discuss how to find such a function.

Consider the second-order nonlinear model in Eq. (1). From the same procedure in the subsection III.1 based on Eq. (13), the sliding motion on the chattering attenuation conventional sliding manifold is enforced by 


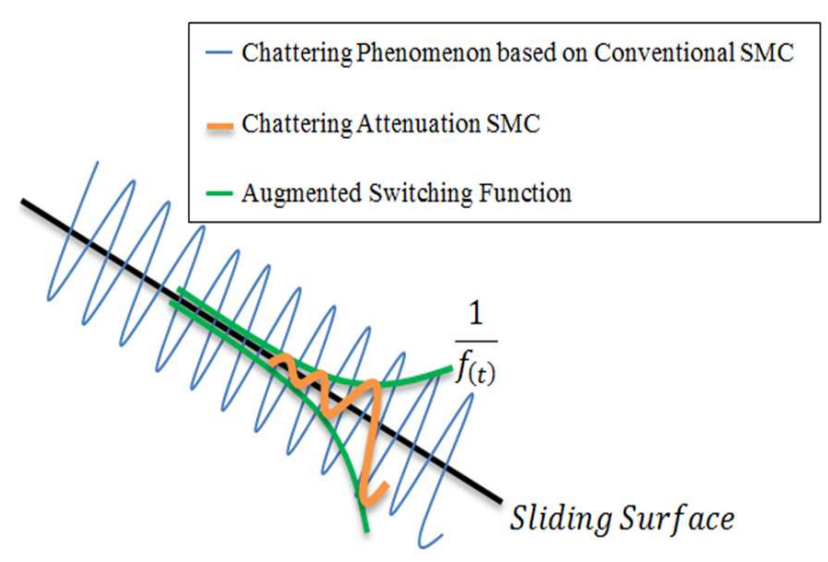

Fig. 1: Chattering attenuation using a time-varying function

the control law as

$$
\begin{aligned}
u=-h(x, \dot{x})^{-1}[ & \mu \frac{\operatorname{sign}\left(\sigma_{C A C S M C}\right)}{f(t)}+\frac{\dot{f}(t)}{f(t)} \sigma_{\text {conv }} \\
& +\lambda \dot{\tilde{x}}+g(x, \dot{x})]
\end{aligned}
$$

Now, the main effect of the sliding manifold including a time-varying function can be described in Remark 3

Remark 3. To suppress the chattering effect, Eq. (14) implies that the value of the signum function should be decreased using the following simple assumption

$$
\lim _{t \rightarrow+\infty} f(t)=+\infty \quad \text { and } \quad f(t) \neq 0
$$

As shown in Eq. (15), to alleviate chattering, the function $f(t)$ is monotonically increasing by time such as an exponential function, logarithmic function, and power function. As an example, assume that $f(t)=\exp (a t+b)$, where $a$ and $b$ are positive constants. Therefore, according to Eq. 14, chattering suppression around the sliding manifold can be displayed in Fig. 1 .

It is observed from Fig. 1 that the chattering effect is reduced exponentially over time for $f(t)=\exp (a t+b)$.
Remark 4. Equation 13 magnifies a scale of the conventional sliding surface with $f(t)$. That implies $\sigma_{\text {conv. }}$ is virtually magnified with $f(t)$, and the Lyapunov's stability must be satisfied for the virtually expanded $\sigma_{c o n v}$. over the time. Thus, the original $\sigma_{c o n v}$. must be reduced to $\frac{1}{f(t)}$. Figure 1 schematically describes a phenomenon whereby the proposed sliding surface changes the scale of the conventional sliding manifold by utilizing the time-varying function $f(t)$ resulting in a new scale afterwards.

Basically, the convergence rate of CSMC is extremely low because the tracking error on the conventional sliding surface reaches zero over an infinite convergence time. To verify this, one can estimate the convergence time $\left(t_{c}\right)$ in the sliding phase in CSMC as follows:

$$
\sigma_{\text {conv } .}=\frac{\mathrm{d} \tilde{x}}{\mathrm{~d} t}+\lambda \tilde{x}=0
$$

where

$$
\frac{\mathrm{d} \tilde{x}}{\tilde{x}}=-\lambda \mathrm{d} t \Rightarrow t_{c}=\left|\frac{\ln \tilde{x}}{\lambda}\right|
$$

To obtain finite time convergence, TSMC is selected in the next subsection.

\section{II.3. Terminal SMC (TSMC) and Non-singular Terminal SMC (NTSMC)}

Nonlinear sliding manifolds for TSMC and NTSMC can improve the performance of CSMC by assuring fast, finite time convergence. Without loss of generality, the terminal sliding manifold is represented as follows:

$$
\sigma_{T S M C}(x, t)=\dot{\tilde{x}}+\lambda \tilde{x}^{\frac{p}{q}} \quad \text { for } \quad 0<p<q
$$


where $p$ and $q$ should be positive odd integers. The nonlinearity term $\tilde{x}^{\frac{p}{q}}$ in Eq. (18) improves the convergence toward an equilibrium point. The finite convergence time $\left(t_{c}\right)$ on the sliding surface can be determined as follows:

$$
\sigma_{T S M C}=\frac{\mathrm{d} \tilde{x}}{\mathrm{~d} t}+\lambda \tilde{x}^{\frac{p}{q}}=0
$$

Then

$$
\frac{\mathrm{d} \tilde{x}}{\tilde{x}^{\frac{p}{q}}}=-\lambda \mathrm{d} t \Rightarrow t_{c}=\frac{q}{\lambda(q-p)}|\tilde{x}(0)|^{\left(1-\frac{p}{q}\right)}
$$

In the same manner as was done with Eq. (6), using the second method of Lyapunov to ensure the stability of the system, let us define a Lyapunov candidate as $V=$ $\frac{1}{2} \sigma_{T S M C}{ }^{2}$. Differentiating $V$ with respect to time results in

$$
\begin{aligned}
\dot{V} & =\sigma_{T S M C} \frac{\partial \sigma_{T S M C}}{\partial t} \\
& =\sigma_{T S M C}\left(\ddot{\tilde{x}}+\lambda \frac{p}{q} \dot{\tilde{x}} \tilde{x}^{\left(\frac{p}{q}-1\right)}\right)
\end{aligned}
$$

Then, to assure the Lyapunov stability the following relation must be satisfied

$$
\ddot{\tilde{x}}+\lambda \frac{p}{q} \dot{\tilde{x}} \tilde{x}^{\left(\frac{p}{q}-1\right)}=-\mu \operatorname{sign}\left(\sigma_{T S M C}\right)
$$

Noticeably, if Eq. (22) satisfies, then we have $\dot{V}=$ $-\mu\left|\sigma_{T S M C}\right|<0$, which can sufficiently guarantee that the state on the terminal sliding surface converges to zero in a finite time. Accordingly, for the nonlinear dynamical system given in Eq. (1), if the terminal sliding surface is chosen as Eq. [18, the terminal sliding mode controller is designed as follows:

$$
\begin{gathered}
u=-h(x, \dot{x})^{-1}\left[\mu \operatorname{sign}\left(\sigma_{T S M C}\right)+\lambda \frac{p}{q} \dot{\tilde{x}} \tilde{x}^{\left(\frac{p}{q}-1\right)}\right. \\
+g(x, \dot{x})]
\end{gathered}
$$

It is clear from Eq. 23 that TSMC cannot ensure a bounded control input for $\dot{\tilde{x}} \neq 0$ when $\tilde{x}=0$ before the states reach the terminal sliding manifold, i.e., TSMC may result in singularity due to a negative value of the fractional power $\left(\frac{p}{q}<1\right)$.

To avoid the singularity problem, NTSMC was adopted as an inverse dynamics in TSMC.

$$
\sigma_{N T S M C}=\tilde{x}+\xi \dot{\tilde{x}}^{\frac{q}{p}} \quad \text { for } \quad 0<p<q<2 p
$$

where Yang et al. [32] proposed that $\xi=\frac{1}{\lambda}$. However, by considering the inverse dynamics strictly, the authors believe that $\xi$ should be chosen as $\left(\frac{1}{\lambda}\right)^{\frac{q}{p}}$. To guarantee that the state trajectory remains around the nonsingular terminal switching surface, the following condition must be satisfied.

$$
\sigma_{N T S M C} \frac{\partial \sigma_{N T S M C}}{\partial t}<0
$$

Taking the time-derivative of Eq. 24 along the system dynamics of Eq. (1) results in

$$
\sigma_{N T S M C} \frac{\partial \sigma_{N T S M C}}{\partial t}=\sigma_{N T S M C}\left(\dot{\tilde{x}}+\xi \frac{q}{p} \ddot{\tilde{x}} \dot{\tilde{x}}^{\left(\frac{q}{p}-1\right)}\right)
$$

Then, to ensure the Lyapunov stability, the following relation must be satisfied

$$
\sigma_{N T S M C}\left(\dot{\tilde{x}}+\xi \frac{q}{p} \ddot{\tilde{x}}^{\left(\frac{q}{p}-1\right)}\right)=-\mu\left|\sigma_{N T S M C}\right| \dot{\tilde{x}}^{\left(\frac{q}{p}-1\right)}
$$

Since $q$ and $p$ are positive odd integers, $(q-p)$ is an even 
integer. Therefore, $\dot{\tilde{x}}^{\left(\frac{q}{p}-1\right)}$ is always positive for $\dot{\tilde{x}} \neq 0$. Then, Eq. 26) can be rewritten as follows:

$$
\begin{aligned}
u=-h(x, \dot{x})^{-1} & {\left[\left(\mu \operatorname{sign}\left(\sigma_{N T S M C}\right)+\dot{\tilde{x}}^{\left(2-\frac{q}{p}\right)}\right) \frac{p}{\xi q}\right.} \\
& +g(x, \dot{x})]
\end{aligned}
$$

As can be observed, the fractional power in Eq. 28 is always positive $\left(2-\frac{q}{p}>0\right)$; thus, the singularity does not occur in NTSMC. However, the traditional NTSMC still includes a discontinuous function $\operatorname{sign}\left(\sigma_{N T S M C}\right)$, which may cause chattering.

\section{II.4. Chattering Attenuation NTSMC (CANTSMC)}

In this subsection, a chattering attenuation nonsingular terminal sliding mode manifold is developed for reducing the value of the discontinuous function over time.

$$
\sigma_{C A N T S M C}=f(t) \sigma_{N T S M C}=f(t)\left(\tilde{x}+\xi \dot{\tilde{x}}^{\frac{q}{p}}\right)
$$

Consider the second-order nonlinear model in Eq. (1). Then, based on Eq. 29], a sliding motion on the chattering attenuation nonsingular terminal sliding manifold is enforced by the control law as follows:

$$
\begin{gathered}
u=-h(x, \dot{x})^{-1}\left[\left(\mu \frac{\operatorname{sign}\left(\sigma_{C A N T S M C}\right)}{f(t)}+\frac{\dot{f}(t)}{f(t)} \frac{\sigma_{N T S M C}}{\dot{\tilde{x}}^{\left(\frac{q}{p}-1\right)}}\right.\right. \\
\left.\left.+\dot{\tilde{x}}^{\left(2-\frac{q}{p}\right)}\right) \frac{p}{\xi q}+g(x, \dot{x})\right]
\end{gathered}
$$

It is important to re-emphasize that the chattering attenuation technique can be applied to any type of SMC approach, not only CSMC.
Remark 5. Note that according to Eq. (30), although the fractional exponent $\left(\frac{q}{p}-1\right)$ is positive, the term $\frac{\sigma_{N T S M C}}{\dot{\tilde{x}}^{\left(\frac{q}{p}-1\right)}}$ does not cause a singularity in practice. The reason is expressed in the following. Foremost, remind that $\sigma_{N T S M C}$ is a distance from the sliding manifold and $\dot{\tilde{x}}$ represents the velocity. Then, note that the chattering occurs around the sliding surface. In the vicinity of the sliding surface, chattering has a very high frequency even though its amplitude is quite small. Thus, we can say that $|\dot{\tilde{x}}|>>\left|\sigma_{N T S M C}\right|$ for any moments in the sliding phase, and the singularity is not a problem.

\section{PERFORMANCE ANALYSIS AND DESIGN PROCEDURE}

In this section, two performance aspects of the proposed chattering attenuation method have been considered: 1) chattering suppression, and 2) robustness. Moreover, each of the aspects introduces a methodology to suitably design the chattering attenuation parameters.

\section{III.1. Chattering Suppression Performance}

In the previous section, the chattering attenuation interpretation of NTSMC has been given. It was considered that the new robust switching function $f(t)$ acting on the chattering attenuation control law is to be time-varying, which incorporates the entire conditions given in Eq. 15. As an example, the augmenting function $f(t)$ can be defined as follows:

$$
f(t)=\exp (a t+b)
$$

where $a$ and $b$ are positive or zero constant scalars and are typically restricted by the maximal value $(F)$ of the time- 
varying function as follows:

$$
\exp \left(a t_{s}+b\right) \leq F \Rightarrow a \leq \frac{\ln (F)-b}{t_{s}}
$$

where $t_{s}$ is a specified time that must be equal or larger than the convergence time $\left(t_{c}\right)$ in the sliding mode controller. The proposed chattering attenuation strategy should start when the states reach near the sliding surface because if the proposed strategy starts at the beginning of the control activity, then the system would have lost its robustness when the states arrive around the sliding plane.

Remark 6. To specify the chattering attenuation parameter $a$, the specified time $t_{s}$ should be selected properly. Since the NTSMC law utilizes a finite convergence time, it is easy to define an approximate value for $t_{s}$. To sufficiently attenuate the chattering phenomenon in the sliding phase, $t_{s}$ should be smaller than or equal to $t_{c}$; i.e., $t_{s}=t_{c}$ is a simple solution. The value of $b$ indicates the effect when the chattering attenuation strategy turns on.

For simplicity, $b=0$ is assumed in this paper. Then, the parameter $a$ should satisfy the following relation:

$$
a \leq \frac{\ln (F)}{t_{c}}
$$

Consequently, Eq. 33 shows the necessary condition to attenuate the chattering phenomenon based on the proposed method.

\section{III.2. Robust Performance}

In this subsection, the robust performance of CSMC is analyzed by applying matched disturbances, and this performance is lately compared with that of chattering attenuation CSMC (CACSMC). A secondorder nonlinear dynamical system with disturbance or parameter uncertainty can be described as follows:

$$
\ddot{x}=g(x, \dot{x})+h(x, \dot{x}) u+d(x, t)
$$

where $d(x, t)$ denotes the matched disturbances or uncertainties that are unknown but bounded as $|d(x, t)|<$ $D$. Taking the time-derivative of the Lyapunov candidate given by Eq. (6) along the uncertain system described by Eq. (34, yields

$$
\begin{aligned}
\dot{V} & =\sigma_{\text {conv }}(\ddot{\tilde{x}}+\lambda \dot{\tilde{x}}) \\
& =\sigma_{\text {conv }} .(g(x, \dot{x})+h(x, \dot{x}) u+d(x, t)+\lambda \dot{\tilde{x}})
\end{aligned}
$$

Equation (35) can be rewritten using Eq. (10) in the form of

$$
\begin{aligned}
& \sigma_{\text {conv } .}(g(x, \dot{x})+h(x, \dot{x}) u+d(x, t)+\lambda \dot{\tilde{x}}) \\
& \leq \sigma_{\text {conv }}\left(-\mu \operatorname{sign}\left(\sigma_{\text {conv } .}\right)+D\right)
\end{aligned}
$$

Noticeably, if $\mu>D$ is satisfied, then the Lyapunov stability, $\dot{V}<0$, is sufficiently guaranteed.

The robust performance of CACSMC can then be evaluated by defining the following Lyapunov candidate:

$$
V=\frac{1}{2} \sigma_{C A C S M C^{2}}
$$

Differentiating Eq. 37) with respect to time along the 


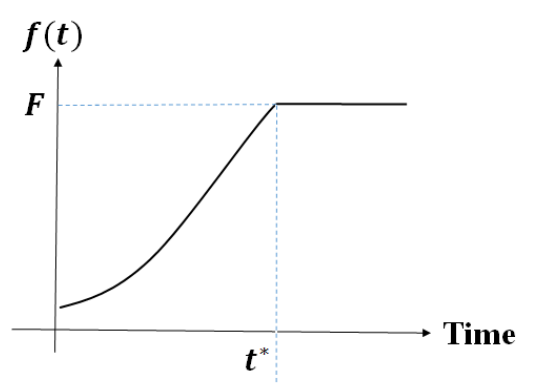

(a)

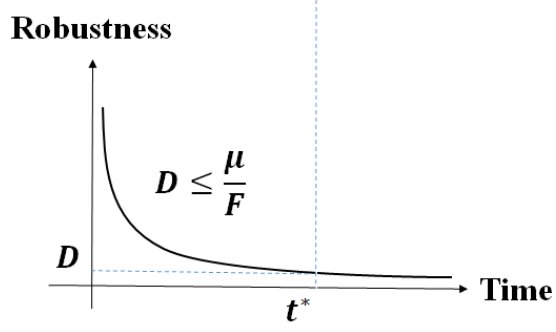

Chattering

(b)

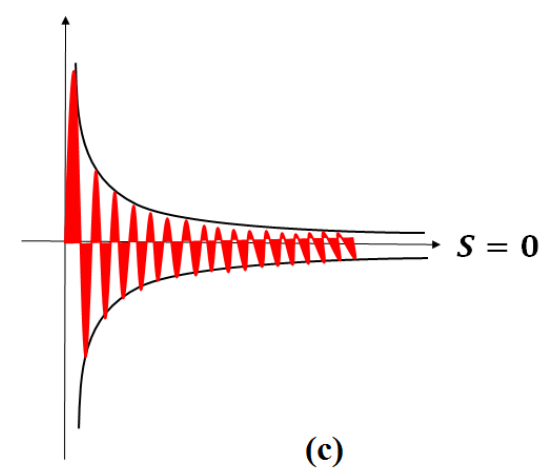

Fig. 2: Schematic performance of the proposed chattering attenuation process with retaining robustness

uncertain system, Eq. (34), leads to

$$
\begin{aligned}
\dot{V}= & \sigma_{C A C S M C}\left[\dot{f}(t) \sigma_{\text {conv } .}+f(t)(\ddot{\tilde{x}}+\lambda \dot{\tilde{x}})\right] \\
= & \sigma_{C A C S M C}\left[\dot{f}(t) \sigma_{\text {conv }}+f(t)(g(x, \dot{x})+h(x, \dot{x}) u\right. \\
& +d(x, t)+\lambda \dot{\tilde{x}})]
\end{aligned}
$$

Thus, substitution of Eq. (14) into Eq. (38) results in

$$
\begin{gathered}
\dot{f}(t) \sigma_{\text {conv } .}+f(t)(g(x, \dot{x})+h(x, \dot{x}) u+d(x, t)+\lambda \dot{\tilde{x}}) \\
\leq-\mu \operatorname{sign}\left(\sigma_{C A C S M C}\right)+f(t) D
\end{gathered}
$$

Clearly, for assuring the Lyapunov stability, the following condition must be satisfied.

$$
\mu \geq|f(t)| D
$$

Eventually, Eq. 40 proves that the robust performance of the proposed chattering attenuation technique can be reduced over time. This is a crucial aspect of the proposed CACSMC approach. To solve this enigmatic problem, one may modify Eq. 15 in Remark 3 as follows:

Remark 7. To suppress the chattering effect as well as retaining the robust performance, $f(t)$ is replaced by an uniformly bounded function as shown in Fig. 2 .

Figure 2 shows the significant effects of the modified chattering attenuation algorithm. The role of the maximal value $F$ is to maintain the robust performance of the system (for $t \geq t^{*}$ ) in an admissible level as well as to attenuate the chattering phenomenon. The admissible level can be defined considering both robust performancekeeping and chattering attenuation. According to Fig. 2 as long as $t<t^{*}$, the switching function is increasing over time. Subsequently, the chattering and the robustness are decreasing over time. Furthermore, for $t \geq t^{*}$, the magnitude of the switching function, the chattering and the robustness are fixed. In practice, this method has several promising attributes:

- The proposed method simply attenuates the chattering phenomenon such that utilizing of the sliding mode controller is feasible in practice. 


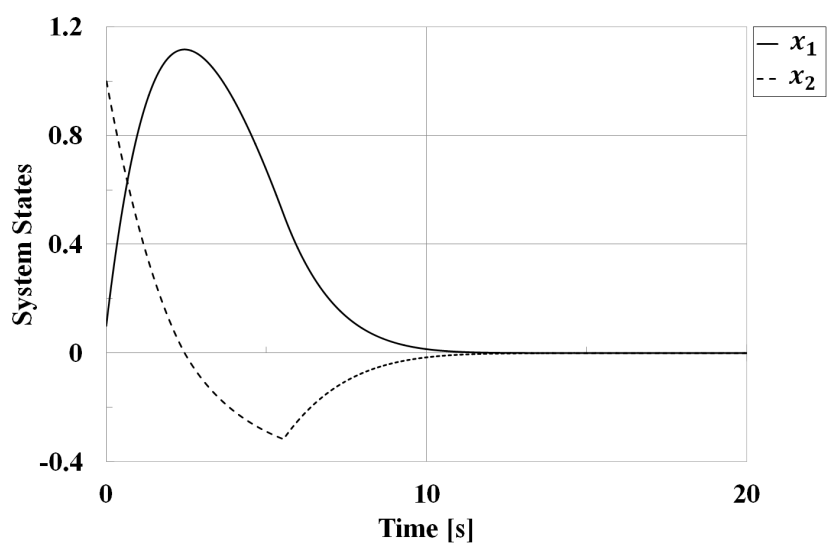

(a)

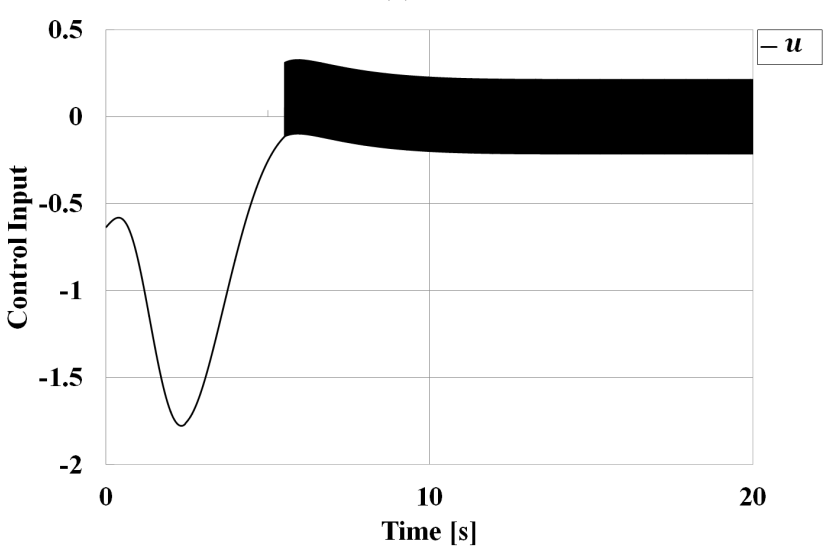

(b)

Fig. 3: Time response of the (a) system states and (b) control input based on NTSMC

- The proposed method retains the robust performance of the controller which can be distinguished from previous methods. In previous algorithms, the continuous approximation of the signum function was employed to eliminate the chattering phenomenon. Nevertheless, the previous algorithms cannot assure the finite reaching time to the sliding manifold and the robust performance of the system in the presence of disturbances or parameter variations.

- The proposed method is an unique design which depends on the robustness and the switching function. The relation between the robustness

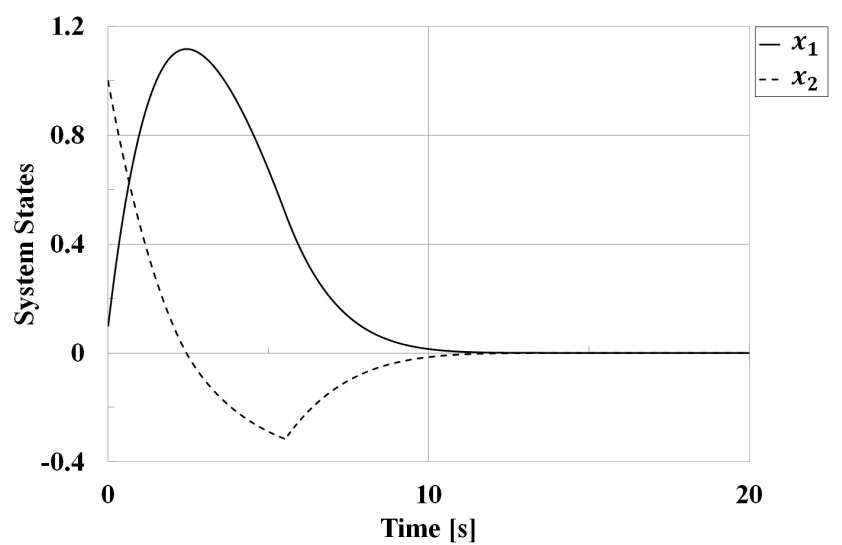

(a)

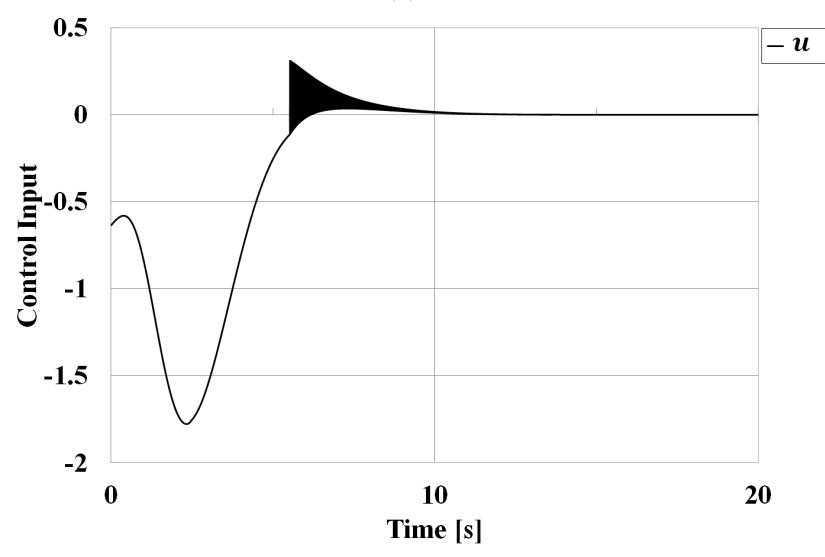

(b)

Fig. 4: Time history of the (a) system states and (b) control input based on CANTSMC with $a=1$ and $b=0$

and the time-varying function corresponds to Eq. (40) $(\mu \geq F \cdot D)$. In the modified chattering attenuation scheme, the chattering phenomenon has been reduced enough and simultaneously the robust performance of the controller can be assured in the presence of disturbances.

Note that one simple method to decrease the chattering phenomenon in a conventional SMC is to employ a small value for the sliding gain $\mu$. However, it implies the robustness of the SMC is reduced. A significant discrepancy between conventional methods and the proposed chattering attenuation technique is apparent for sporadic disturbances. For such cases, the 
maximal value $F$ can be selected as a large value, and the chattering is attenuated sufficiently without reducing the controller's robustness. Furthermore, in the proposed scheme the control strategy can be kept in action any time, because required control inputs approach zero in a short time (see Fig. 7b in Section IV.

\section{SIMULATIONS}

To present the efficacy of the proposed method, this section provides a simulation study of a single-input second order nonlinear system. The system equation is expressed as follows when there is no noise.

$$
\left\{\begin{array}{l}
\dot{x}_{1}=x_{2} \\
\dot{x}_{2}=x_{1}^{4} \cos x_{2}+u
\end{array}\right.
$$

where $x_{1}$ and $x_{2}$ are states of the dynamical system and $u$ is a control input. The initial values of the states are set as $\left[x_{1}(0), x_{2}(0)\right]=[0.1,1]$. The task is to settle the states from the given initial values to zero and keep them stable. According to the explanations in Section II a non-singular terminal sliding surface is selected as

$$
\sigma_{N T S M C}=\tilde{x}_{1}+\xi \tilde{x}_{2}^{\frac{q}{p}}
$$

where the sliding gains are given as $\xi=2$ and $\mu=\frac{1}{2}$. Moreover, the required convergence time of the sliding phase in NTSMC can be determined as follows:

$$
\tilde{x}_{1}+\xi\left(\frac{\mathrm{d} \tilde{x}_{1}}{\mathrm{~d} t}\right)^{\frac{q}{p}}=0 \Rightarrow t_{c}=\frac{q}{q-p} \xi^{\frac{p}{q}}\left|\tilde{x}_{1}(0)^{\left(1-\frac{p}{q}\right)}\right|
$$

Therefore, one can find an admissible value for $(p, q)$ by a given convergence time $\left(t_{c}\right)$. In this simulation, by selecting the convergence time as $t_{c}=4.5(s)$ and using

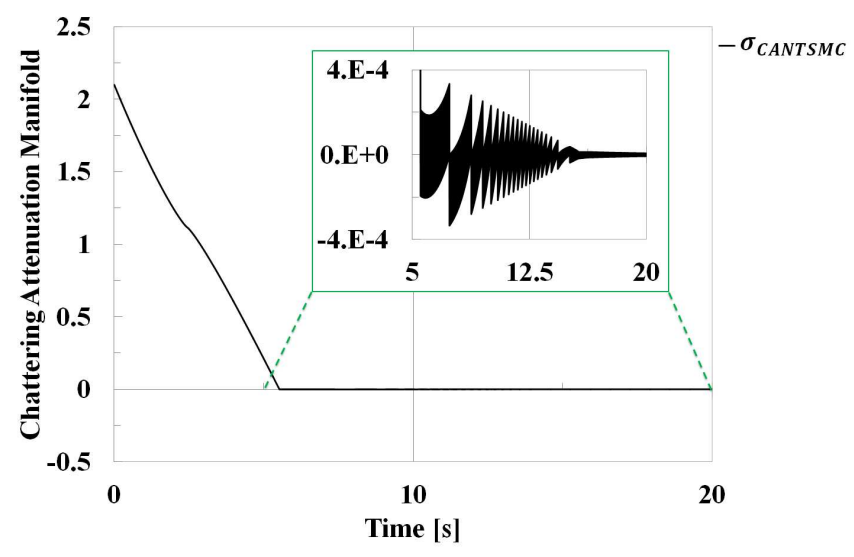

Fig. 5: Time history of the chattering attenuation surface with $a=1$ and $b=0$

Eq. 43, the fractional exponents $(p, q)$ are selected as

$$
p=11 \quad, \quad q=13
$$

Note that instead of power commands in MATLAB, the "nthroot" command should be served for giving the real $n^{t h}$ root of any real numbers.

To investigate the robustness of the proposed control law, the following three cases subject to the disturbances are addressed:

- Case A: Traditional NTSMC subject to a sporadic disturbance,

- Case B: Simulation for a sporadic disturbance based on the CANTSMC with $F=10^{7}$,

- Case C: Simulation for a continuous disturbance based on the CANTSMC with $F=5$ and $D=0.05$

Using the NTSMC law in Eq. 42, the time history of the states are shown in Fig. 3a It can be clearly seen that the states reach the target values within a finite time. The manifestation of the chattering phenomenon induced by having apparent fluctuations in the control input based upon a nonsingular terminal sliding mode controller is shown in Fig. 3b. 


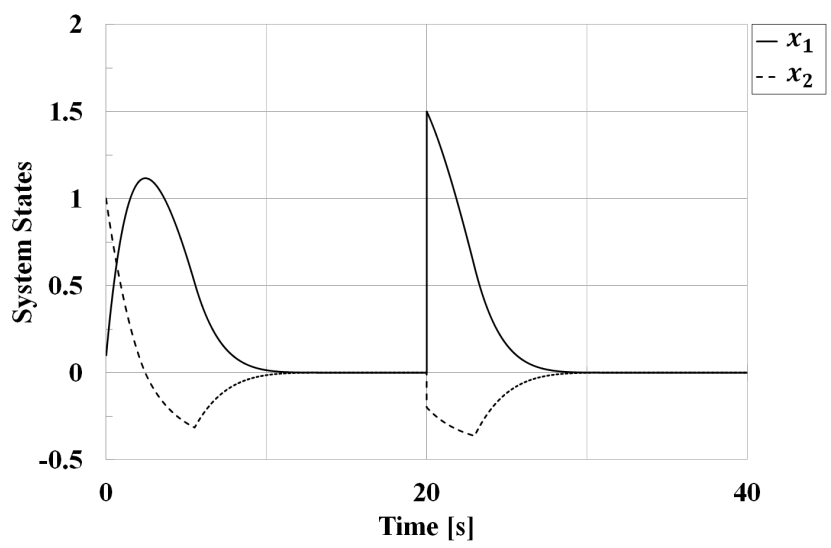

(a)

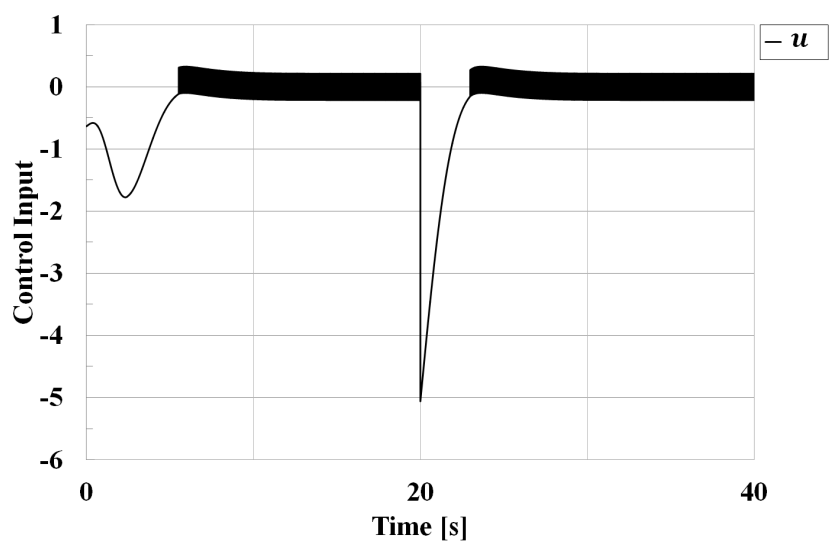

(b)

Fig. 6: Time history of the (a) state variables and (b) control input subject to the sporadic disturbance for Case A

As discussed in Section III the chattering attenuation interpretation of NTSMC was given. It was considered that the new switching function $f(t)$ acting on the chattering attenuation control law is to be time-varying, which incorporates the entire conditions given in Eqs. (31) and 33. The proposed control scheme for robust control of an aforementioned dynamical system in Eq. (41) provides insights for suppressing the chattering effect as well as for maintaining the robust performance by the following equivalent coefficient. Maximum value of the switching function can be assumed as $F=10^{7}$. Then, from Eq. 33,

$$
a \leq 3.58
$$

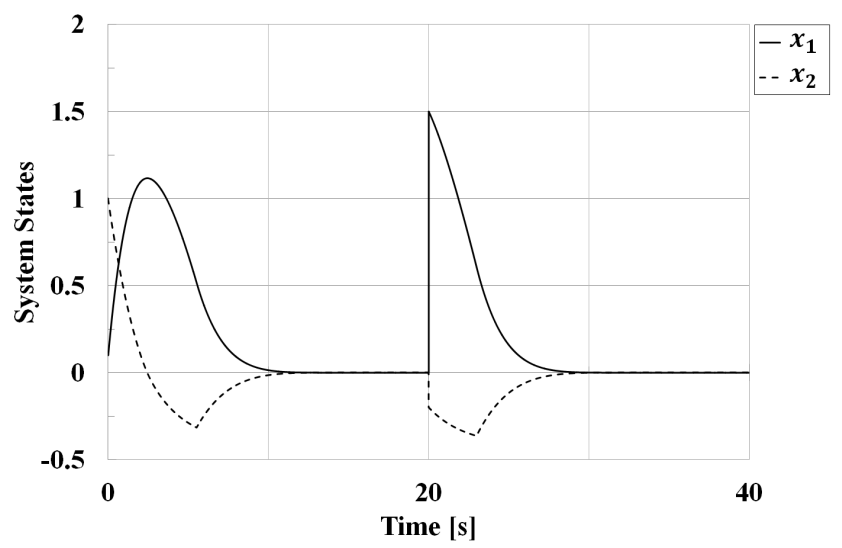

(a)

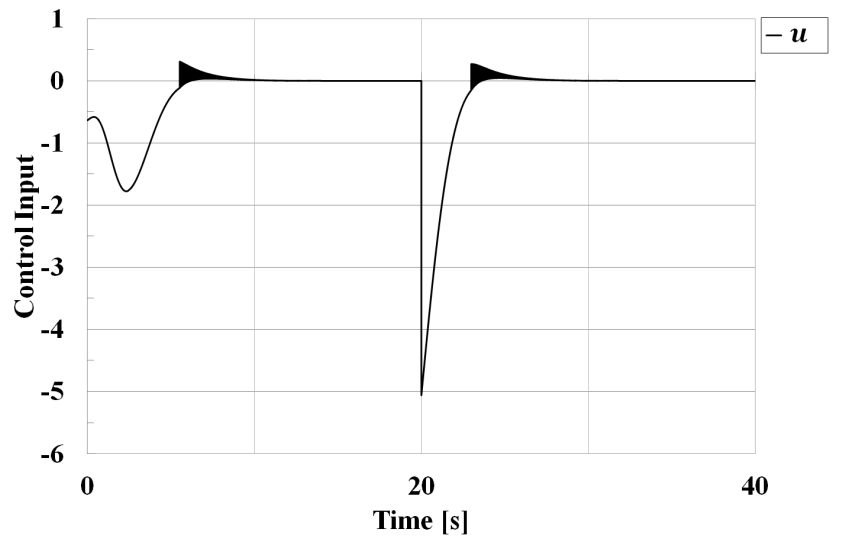

(b)

Fig. 7: Time response of the (a) state variables and (b) control input subject to the sporadic disturbance for Case B

According to Eq. (45), the parameter $a=1$ is selected in this paper.

The simulation results of the CANTSMC method for robust control of the second order nonlinear dynamical system 41, are demonstrated in Fig. 4. Figure $4 a$ illustrates the time variations of the system states. Figure $4 b$ depicts the time history of the control input based on CANTSMC. Time response of the proposed chattering attenuation nonsingular terminal sliding manifold $\sigma_{C A N T S M C}$ has been displayed in Fig. 5. It is observed from Figs. $3 \mathrm{~b}$ and $4 \mathrm{~b}$ that employing CANTSMC can provide chattering attenuation control responses in comparison with the responses obtained by 
employing NTSMC.

As another sporadic disturbance, the second disturbance is exerted at $t=20$ (s) as $\left[x_{1}(20), x_{2}(20)\right]=$ $[1.5,-0.2]$. Time history of the state variables and control input for Case A are demonstrated in Figs. 6a and $6 \mathrm{~b}$, respectively. Figure $6 \mathrm{a}$ represents that the state variables converge to the desired values in the presence of a sporadic disturbance. However, the time response of the corresponding controller is shown in Fig. 6b It is observed that the chattering phenomenon in the control input occurs based on the conventional NTSMC method which can waste the control energy. Time response of the system's states and control input are displayed in Figs. 7a and $7 \mathrm{~b}$ respectively for Case B. Figure $7 \mathrm{~b}$ presents that the control input becomes almost zero at 10 (s) $<t<20$ (s) and $t>30$ (s) even though the CANTSMC is active. Accordingly, Fig. 8 illustrates the time history of the proposed chattering attenuation manifold in the presence of the sporadic disturbance.

For Case $\mathbf{C}$, the random noise $d(x, t)$ with $|d(x, t)| \leq 0.05$ is added into the second relation of Eq. (41). Then, to satisfy Eq. (40, $F$ can be specified as 5; i.e.,

$$
\mu \geq|f(t)| D \quad \Rightarrow \quad 0.5 \geq 5 \times 0.05=0.25
$$

Figures $9 \mathrm{a}$ and $9 \mathrm{~b}$ depict the time variations of the system states and the control input, respectively for Case C. As can be seen from Fig. 9b, the chattering has been alleviated by means of the upper bound of time-varying function to keep the robust performance of the control system in the presence of a continuous disturbance. Time response of the proposed chattering attenuation manifold has been displayed in Fig. 10 subject to the continuous

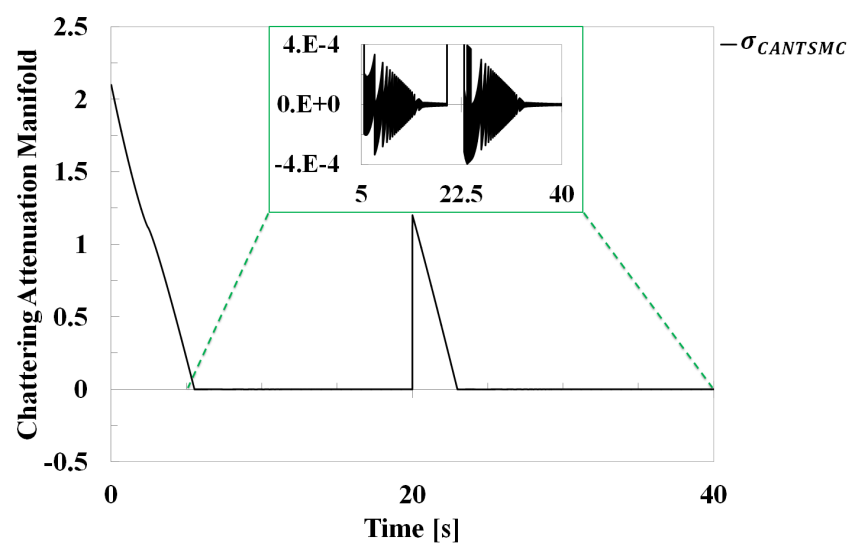

Fig. 8: Time history of the chattering attenuation surface for Case B

random disturbance.

Note that the chattering attenuation method shows similar time histories of state variables with conventional ones for continuous random disturbances. However, the effectiveness of the proposed chattering attenuation approach is obviously seen for sporadic disturbances as shown in Case A.

\section{CONCLUSION}

In this study, an effective implementation of a new approach for controlling nonlinear systems has been developed on the basis of a chattering attenuation nonsingular terminal sliding mode control (CANTSMC) technique. The proposed control law includes a new time-varying switching function that can reduce the value of discontinuous functions over time. Usually, there is a delicate balance between chattering and robustness. However, by suitably selecting the control parameters in the CANTSMC method, controller responses become much more insensitive to parameter uncertainty and disturbances in comparison to the response obtained by the non-singular terminal sliding mode control (NTSMC) 


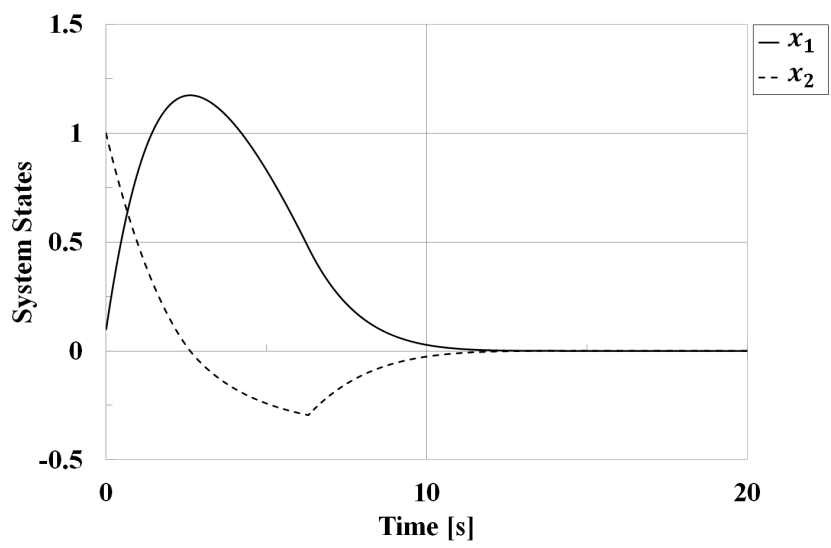

(a)

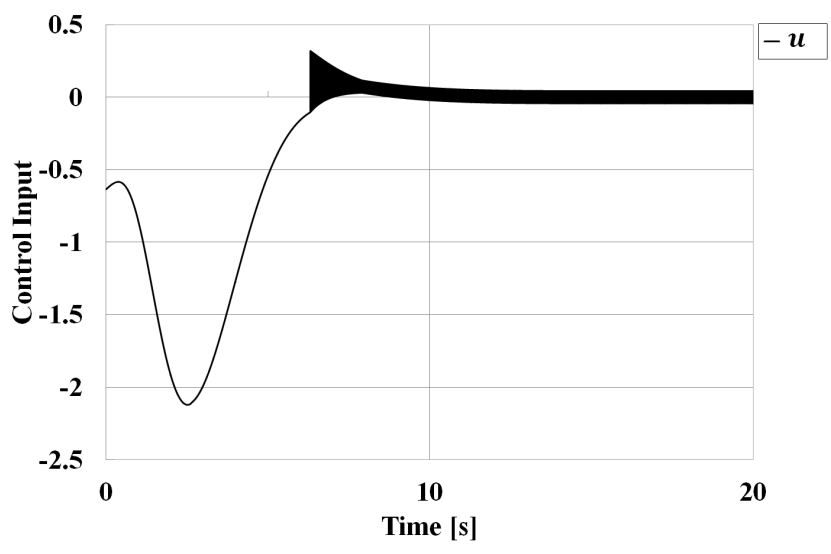

(b)

Fig. 9: Time history of the (a) state variables and (b) control input subject to the continuous random disturbance for Case $\mathrm{C}$

method. On the other hand, the robustness of the system can be further improved by using the appropriate CANTSMC method. In addition, it can be emphasized that the proposed chattering attenuation technique can be applied to any type of sliding mode approach, not only traditional NTSMC methods. The simulation results for a single-input second order nonlinear dynamical system showed that the new sliding surface and associated control law are effective in chattering attenuation of control input and robust performance against disturbances.

\section{REFERENCES}

1. Utkin, V. I., Sliding Modes in Control and

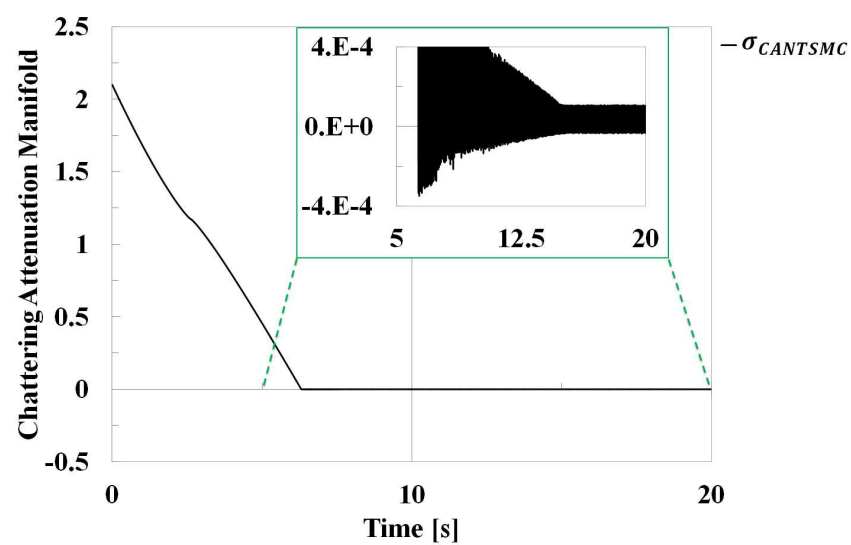

Fig. 10: Time history of the chattering attenuation surface subject to the continuous random disturbance for Case $\mathrm{C}$

Optimization, Springer, New York (1992).

2. Corradini, M. L., V. Fossi, A. Giantomassi, G. Ippoliti, S. Longhi, and G. Orlando, "Discrete time sliding mode control of robotic manipulators: development and experimental validation", Control Engineering Practice, Vol. 20, No. 8, pp. 816-822 (2012). doi: 10.1016/j.conengprac.2012.04.005

3. Plestan, F., Y. Shtessel, V. Brégeault, and A. Poznyak, "Sliding mode control with gain adaptation-Application to an electropneumatic actuator", Control Engineering Practice, Vol. 21, No. 5, pp. 679-688 (2013). doi: 10.1016/j.conengprac.2012.04.012

4. Fridman, L., J. Moreno, and R. Iriarte, Sliding Modes after the First Decade of the $21^{\text {st }}$ Century, Springer, Berlin Heidelberg (2011).

5. Yu, X., and J.-X. Xu, Variable Structure Systems: Towards the $21^{\text {st }}$ Century, Springer, Berlin Heidelberg (2002).

6. Slotine, J. J., and S. S. Sastry, “Tracking control of nonlinear systems using sliding surfaces, with application to robot manipulators", International Journal of Control, Vol. 38, No. 2, pp. 465-492 (1983). 
doi: $10.1080 / 00207178308933088$

7. Lu, K., Y. Xia, Z. Zhu, and M. V. Basin, "Sliding mode attitude tracking of rigid spacecraft with disturbances", Journal of the Franklin Institute, Vol. 349, No. 2, pp. 413-440 (2012).

doi: 10.1016/j.jfranklin.2011.07.019

8. Harl, N., and S. N. Balakrishnan, "Reentry terminal guidance through sliding mode control”, Journal of Guidance, Control, and Dynamics, Vol. 33, No. 1, pp. 186-199 (2010).

\section{doi: $10.2514 / 1.42654$}

9. Sumantr, B., N. Uchiyama, and S. Sano, "Least square based sliding mode control for a quad-rotor helicopter and energy saving by chattering reduction", Mechanical Systems and Signal Processing, Vol. 6667, pp. 769-784 (2016).

doi: 10.1016/j.ymssp.2015.05.013

10. Wang, J.-M., J.-J. Liu, B. Ren, and J. Chen, "Sliding mode control to stabilization of cascaded heat PDEODE systems subject to boundary control matched disturbance", Automatica, Vol. 52, No. 1, pp. 23-34 (2015).

doi: 10.1016/j.automatica.2014.10.117

11. Al-Hadithi, B. M., A. J. Barragan, J. M. Andujar, and A. Jimenez, "Variable structure control with chattering elimination and guaranteed stability for a generalized T-S model", Applied Soft Computing, Vol. 13, No. 12, pp. 4802-4812 (2013).

doi: 10.1016/j.asoc.2013.07.026

12. Emel'yanov, S. V., S. K. Korovin, and A. Levant, "High-order sliding modes in control systems", Computational Mathematics and Modeling, Vol. 7, No. 3, pp. 294-318 (1996). doi: $10.1007 / \mathrm{BF} 01128162$
13. Levant, A., "Higher-order sliding modes, differentiation and output feedback control", International Journal of Control, Vol. 76 (9/10), pp. 924-941 (2003). doi: $10.1080 / 0020717031000099029$

14. Tournes, C., and Y. Shtessel, "Automatic docking using optimal control and second order sliding mode control", AIAA Guidance, Navigation and Control Conference and Exhibit, South Carolina, 20-23 Aug. (2007).

\section{doi: $10.2514 / 6.2007-6345$}

15. Defoort, M., T. Floquet, A. Kokosy, and W. Perruquetti, "A novel higher order sliding mode control scheme", Systems \& Control Letters, Vol. 58, No. 2, pp. 102-108 (2009).

doi: 10.1016/j.sysconle.2008.09.004

16. Li, J., Y. Pan, and K. D. Kumar, "Design of asymptotic second-order sliding mode control for satellite formation flying", Journal of Guidance, Control, and Dynamics, Vol. 35, No. 1, pp. 309-316 (2012).

doi: $10.2514 / 1.55747$

17. Yamasaki, T., S. N. Balakrishnan, and H. Takano, "Separate-channel integrated guidance and autopilot for automatic path-following”, Journal of Guidance, Control, and Dynamics, Vol. 36, No. 1, pp. 25-34 (2013).

\section{doi: $10.2514 / 1.58259$}

18. Furfaro, R., D. Cersosimo, and D. R. Wibben, "Asteroid precision landing via multiple sliding surfaces guidance techniques", Journal of Guidance, Control, and Dynamics, Vol. 36, No. 4, pp. 1075-1092 (2013).

doi: $10.2514 / 1.58246$

19. Feng, Y., F. Han, and X. Yu, "Chattering free full-order 
sliding-mode control", Automatica, Vol. 50, No. 4, pp. 1310-1314 (2014).

doi: 10.1016/j.automatica.2014.01.004

20. Boiko, I., L. Fridman, and M. I. Castellanos, "Analysis of second-order sliding mode algorithms in the frequency domain", IEEE Trans. on Automatic Control, Vol. 49, No. 6, pp. 946-950 (2004). doi: 10.1109/TAC.2004.829615

21. Boiko, I. M., "On relative degree, chattering and fractal nature of parasitic dynamics in sliding mode control", Journal of the Franklin Institute, Vol. 351, No. 4, pp. 1939-1952 (2014).

doi: 10.1016/j.jfranklin.2013.01.003

22. Yu, S., X. Yu, B. Shirinzadeh, and Z. Man, "Continuous finite-time control for robotic manipulators with terminal sliding mode", Automatica, Vol. 41, No. 11, pp. 1957-1964 (2005). doi: 10.1016/j.automatica.2005.07.001

23. Venkataraman, S. T., and S. Gulati, "Control of nonlinear systems using terminal sliding modes", Journal of Dynamic Systems, Measurement, and Control, Vol. 115, No. 3, pp. 554-560 (1993). doi: $10.1115 / 1.2899138$

24. Feng, Y., X. Yu and, Z. Man, "Non-singular terminal sliding mode control of rigid manipulators", Automatica, Vol. 38, No. 12, pp. 2159-2167 (2002). doi: 10.1016/S0005-1098(02)00147-4

25. Lin, C.-K., "Nonsingular terminal sliding mode control of robot manipulators using fuzzy wavelet networks", IEEE Trans. on Fuzzy Systems, Vol. 14, No. 6, pp. 849859 (2006).

doi: 10.1109/TFUZZ.2006.879982

26. Komurcugil, H., "Non-singular terminal slidingmode control of DCDC buck converters", Control
Engineering Practice, Vol. 21, No. 3, pp. 321-332 (2013).

doi: 10.1016/j.conengprac.2012.11.006

27. Bayramoglu, H., and H. Komurcugil, "Nonsingular decoupled terminal sliding mode control for a class of fourth-order nonlinear systems", Communications in Nonlinear Science and Numerical Simulation, Vol. 18, No. 9, pp. 2527-2539 (2013).

doi: 10.1016/j.cnsns.2012.11.008

28. Slotine, J.-J. E., and W. P. Li, Applied Nonlinear Control: Chap. 7 (Sliding Control), Prentice Hall, Englewood cliffs, NJ (1991).

29. Azar, A. T., and Q. Zhu, Advances and Applications in Sliding Mode Control Systems, Springer, Switzerland, (2015).

30. Moreno, J. A., "On strict Lyapunov functions for some non-homogeneous super-twisting algorithms", Journal of the Franklin Institute, Vol. 351, No. 4, pp. 1902-1919 (2014).

doi: 10.1016/j.jfranklin.2013.09.019

31. Kunusch, C., P. Puleston, and M. Mayoski, Sliding Mode Control of PEM Fuel Cells, Springer-Verlag, London, (2012).

32. Yang, J., S. Li, J. Su, and X. Yu, "Continuous nonsingular terminal sliding mode control for systems with mismatched disturbances", Automatica, Vol. 49, No. 7, pp. 2287-2291 (2013).

doi: 10.1016/j.automatica.3013.03.026 